\title{
First VLTI/MIDI observations of a Be star: Alpha Arae ${ }^{\star}$
}

\author{
O. Chesneau ${ }^{1,2}$, A. Meilland ${ }^{2}$, T. Rivinius ${ }^{3}$, Ph. Stee ${ }^{2}$, S. Jankov ${ }^{4}$, A. Domiciano de Souza ${ }^{5}$, U. Graser ${ }^{1}$, T. Herbst ${ }^{1}$, \\ E. Janot-Pacheco ${ }^{6}$, R. Koehler ${ }^{1}$, C. Leinert ${ }^{1}$, S. Morel ${ }^{7}$, F. Paresce ${ }^{7}$, A. Richichi ${ }^{7}$, and S. Robbe-Dubois ${ }^{4}$ \\ ${ }^{1}$ Max-Planck-Institut für Astronomie, Königstuhl 17, 69117 Heidelberg, Germany \\ e-mail: Olivier.Chesneau@obs-azur.fr \\ 2 Observatoire de la Côte d'Azur, CNRS UMR 6203, Avenue Copernic, Grasse, France \\ 3 Landessternwarte Heidelberg, Königstuhl 12, 69117 Heidelberg, Germany \\ ${ }^{4}$ Laboratoire Universitaire d'Astrophysique de Nice, France \\ 5 Max-Planck-Institut für Radioastronomie, Auf dem Hügel 69, 53121 Bonn, Germany \\ 6 Instituto de Astronomia, Geofiśica e Ciências Atmosféricas da Universidade de São Paulo (IAG-USP), \\ CP 9638, 01065-970 São Paulo, Brazil \\ ${ }^{7}$ European Southern Observatory, Karl-Schwarzschild-Strasse 2, 85748 Garching, Germany
}

Received 6 September 2004 / Accepted 5 January 2005

\begin{abstract}
We present the first VLTI/MIDI observations of the Be star alpha Ara (HD 158 427), showing a nearly unresolved circumstellar disk in the $N$ band. The interferometric measurements made use of the UT1 and UT3 telescopes. The projected baselines were 102 and 74 meters with position angles of $7^{\circ}$ and $55^{\circ}$, respectively. These measurements put an upper limit on the envelope size in the $N$ band under the uniform disk approximation of $\phi_{\max }=4 \pm 1.5$ mas, corresponding to $14 R_{\star}$, assuming $R_{\star}=4.8 R_{\odot}$ and the Hipparcos distance of $74 \mathrm{pc}$.

On the other hand the disk density must be large enough to produce the observed strong Balmer line emission. In order to estimate the possible circumstellar and stellar parameters we have used the SIMECA code developed by Stee et al. (1995, A\&A, 300, 219) and Stee \& Bittar (2001, A\&A, 367, 532). Optical spectra taken with the échelle instrument HEROS and the ESO-50 cm telescope, as well as infrared ones from the 1.6m Brazilian telescope were used together with the MIDI spectra and visibilities. These observations place complementary constraints on the density and geometry of the alpha Ara circumstellar disk. We discuss the potential truncation of the disk by a companion and we present spectroscopic indications of a periodic perturbation of some Balmer lines.
\end{abstract}

Key words. techniques: high angular resolution - techniques: interferometric - stars: emission-line, $\mathrm{Be}-$ stars: winds, outflows - stars: individual: $\alpha$ Ara - circumstellar matter

\section{Introduction}

Be stars are hot stars exhibiting the so-called Be phenomenon, i.e. Balmer lines in emission and infrared excess. Optical/infrared and ultraviolet observations of Be stars have been widely interpreted as evidence for two quite distinct regions in the circumstellar environment of these objects: a rotating, dense equatorial region, called the "disk" in the following, and a diluted polar region which expands with velocities that may reach $2000 \mathrm{~km} \mathrm{~s}^{-1}$ (e.g. Marlborough 1986).

The thermal infrared domain is an important spectral region where the transition between the optically thin and thick disk occurs. The optically thick disk emission increases from 7 to $15 \mu \mathrm{m}$. It reaches about half of the total continuum flux at $8 \mu \mathrm{m}$ and dominates the Spectral Energy Distribution (SED) almost completely at 15-20 $\mu \mathrm{m}$ (Gehrz et al. 1974). The flux from the central star at these wavelengths is 10 to 50 times fainter

* Based on observations made with the Very Large Telescope Interferometer at Paranal Observatory. compared to $\lambda=2 \mu \mathrm{m}$ whereas the ratio $F_{\text {env }} / F_{\star}$ reaches a factor of 4-10. In particular for nearly edge-on disks, the flux from the optically thick disk is very sensitive to the inclination angle, since it is proportional to the emitting surface.

Observations at $20 \mu \mathrm{m}$ by Gehrz et al. (1974) demonstrated that the mid-IR excess is due to free-free emission from the hydrogen envelope. The ad-hoc model from Waters (1986) has been successful explaining near- and far-IR observations and is coherent with polarization data (Coté \& Waters 1987; Waters \& Marlborough 1992; Dougherty et al. 1994; Coté et al. 1996). In this model the IR excess originates from a disk with an opening angle $\theta$ and a density distribution $\rho(r) \propto r^{-n}$ seen at an observer's angle $i$. From IRAS data of four Be stars Waters found that the far-IR slope of the SED is not strongly influenced by the opening angle, as long as $\theta+i \leq 90^{\circ}$. Moreover, he found that $n=2.4$ gives a good agreement for $\chi$ Oph, $\delta$ Cen and $\phi$ Per if the disk is truncated to $6.5 R_{*}$.

Interferometry in the visible has also been used to study the circumstellar environment of Be stars (Thom et al. 1986; 
Mourard et al. 1989; Quirrenbach et al. 1994; Stee et al. 1995). Quirrenbach et al. (1997) gave an upper limit on the opening angle of about $20^{\circ}$ and using spectral Differential Interferometry (DI), structures within Be disks were monitored with a high spatial resolution over several years (Vakili et al. 1998; Berio et al. 1999). Nevertheless, the disk extension at IR wavelengths is still subject of an active debate since various authors give quite different extensions as a function of wavelength. For instance Waters (1986) found a typical IR extension of $16 R_{\star}$ from 12, 25 and $60 \mu$ m observations, Gehrz et al. (1974) found $8 R_{\star}$ at 2.3 and $19.5 \mu \mathrm{m}$, Dougherty et al. (1994) a disk size larger than $20 R_{\star}$ in the near-IR, and finally Stee $\&$ Bittar (2001) obtained that the Br $\gamma$ emission line and the nearby continuum originates from a very extended region with a typical size of $40 R_{\star}$.

In order to solve this issue, we have used the VLTI/MIDI interferometer to obtain the first IR measurements in the $N$ spectral band of a Be star. The selected target, alpha Ara (HD 158 427, HR 6510, B3 Ve), is one of the closest Be stars with an estimated distance of $74 \pm 6 \mathrm{pc}$ derived from the Hipparcos parallax, and is well known as an emission line star since the discovery of its $\mathrm{H} \beta$ emission by Pickering (1896, 1896). Alpha Ara is a $2.9 \mathrm{mag}$ star in the Johnson $V$-band and its IRAS flux at 12 micron is $12.7 \mathrm{Jy}$. Its color excesses $E(V-L)$ and $E(V-12 \mu \mathrm{m})$ are respectively 1.8 and 2.23 , being among the highest of its class. The projected rotational velocity $v \sin i$ has been estimated to be $250-300 \mathrm{~km} \mathrm{~s}^{-1}$ (Yudin 2001; Chauville et al. 2001). An intrinsic linear polarization of $\mathrm{Pl} \approx 0.6 \%$ with a Position Angle (PA) of $172^{\circ}$ has also been detected (McLean \& Clarke 1979; Yudin 2001). Since the disk orientation is expected to be perpendicular to this direction, we expect the disk major-axis to be around $\mathrm{PA} \approx 82^{\circ}$ (Wood et al. 1996a; Quirrenbach et al. 1997).

The spectral type definition of alpha Ara is constant in the literature ranging from B2 Ve to B3 Ve. We adopted B3 Ve following the latest study of Chauville et al. (2001). The typical stellar radius and effective temperature for this spectral type are $4.8 R_{\odot}$ and $T_{\text {eff }}=18000$ respectively.

By using IRAS observations of alpha Ara, Waters (1986) estimated the outer disk radius is estimated as about $7 R_{\star}$, i.e. 2.1 mas at a distance of $74 \mathrm{pc}$. Stee (2003) predicts the visibility of alpha Ara at $2 \mu \mathrm{m}$ to be lower than 0.2 with a $60 \mathrm{~m}$ baseline, i.e. fully resolved. Using the same model parameters, a significant visibility loss should still be detectable in the $N$ band. This paper will show that at $10 \mu \mathrm{m}$ alpha Ara is in fact unresolved which, combined with spectroscopic data, puts some constraints on density, inclination angle, disk orientation and distance of alpha Ara.

The paper is organized as follows. In Sect. 2 we present the interferometric MIDI observations and the $H$-band spectroscopy. Section 3 briefly describes the SIMECA code and the envelope parameters used for the modeling of alpha Ara. The modeling strategy is described in detail. In Sect. 4 we present our theoretical results and discuss the possibility for a close but unseen companion to truncate the disk making alpha Ara unresolved in the $N$ band. Section 5 presents some spectroscopic variations of the $\mathrm{H} \beta$ line profile that may be further evidence of this hypothetical companion. Finally, Sect. 6 draws the main
Table 1. Journal of observations.

\begin{tabular}{llcr}
\hline \hline \multicolumn{4}{c}{ June, 16, 2003 night, $B=102 \mathrm{~m}, \mathrm{PA}=7^{\circ}$} \\
\hline Star & Template & \multicolumn{1}{c}{ Time } & Frames \\
\hline HD 165 135 & tracking & $00: 36: 11$ & 15000 \\
HD 165 135 & phot. UT1 & $00: 42: 33$ & 2000 \\
HD 165 135 & phot. UT3 & $00: 44: 12$ & 2000 \\
HD 152 786 & tracking & $01: 06: 42$ & 12000 \\
HD 152 786 & phot. UT1 & $01: 12: 15$ & 3000 \\
HD 152 786 & phot. UT3 & $01: 14: 33$ & 3000 \\
$\alpha$ Ara & tracking & $01: 35: 27$ & 12000 \\
$\alpha$ Ara & phot. UT1 & $01: 40: 55$ & 3000 \\
$\alpha$ Ara & phot. UT3 & $01: 43: 12$ & 3000 \\
HD 165 135 & tracking & $02: 46: 05$ & 12000 \\
HD 165 135 & phot. UT1 & $02: 51: 32$ & 3000 \\
HD 165 135 & phot. UT3 & $02: 46: 05$ & 3000 \\
$\alpha$ Aq1 & tracking & $06: 44: 35$ & 12000 \\
$\alpha$ Aql & phot. UT1 & $06: 50: 08$ & 3000 \\
$\alpha$ Aq1 & phot. UT3 & $06: 52: 14$ & 3000 \\
\hline \multicolumn{1}{c}{ June, 17, 2003 night, $B=79 \mathrm{~m}$, PA $=55^{\circ}$} \\
\hline HD 139 997 & tracking & $05: 31: 17$ & 10000 \\
HD 139 997 & phot. UT1 & $05: 35: 38$ & 2000 \\
HD 139 997 & phot. UT3 & $05: 37: 29$ & 2000 \\
HD 168 545 & tracking & $06: 35: 05$ & 15000 \\
HD 168 545 & phot. UT1 & $06: 40: 33$ & 2000 \\
HD 168 545 & phot. UT3 & $06: 42: 21$ & 2000 \\
$\alpha$ Ara & tracking & $07: 09: 19$ & 12000 \\
$\alpha$ Ara & phot. UT1 & $07: 14: 47$ & 3000 \\
$\alpha$ Ara & phot. UT3 & $07: 17: 05$ & 3000 \\
\hline
\end{tabular}

conclusions from these first IR interferometric measurements of a Be star.

\section{Observations}

\subsection{Interferometric data}

We use the VLTI/MIDI Interferometer described by Leinert et al. (2003a,b) which combines the mid-IR light ( $N$ band) from the two VLT Unit Telescopes Antu (UT1) and Melipal (UT3). The observations of alpha Ara were done during the nights of June, 16th and 17th 2003 and are presented in Table 1.

On the 16th of June 2003, alpha Ara was observed with a $102 \mathrm{~m}$ baseline and a PA $=7^{\circ}$; on June, 17 the projected baseline was $79 \mathrm{~m}$ and $\mathrm{PA}=55^{\circ}$.

The observing sequence described extensively in Przygodda et al. (2003) is summarized hereafter. The chopping mode $\left(f=2 \mathrm{~Hz}\right.$, angle $\left.-90^{\circ}\right)$ is used to point and visualize the star. The number of frames recorded by acquisition file are generally 2000, the exposure time is by default $4 \mathrm{~ms}$ in order to avoid background saturation. If the result of the acquisition sequence is not satisfactory, this procedure is re-executed. Then, the MIDI beam combiner, the wide slit $\left(0 .{ }^{\prime} 6 \times 2^{\prime \prime}\right)$, and the $\mathrm{NaCl}$ prism are inserted to disperse the light and search 
for the fringes by moving the VLTI delay lines. The resulting spectra have a resolution $\lambda / \Delta \lambda \sim 30$.

When searching for the fringe signal, the delay line of the VLTI is moved, while the MIDI internal piezo-driven delay line performs additional scans of the fringe pattern. Once the fringes are found another file is recorded while MIDI tracks the fringes on its own, i.e. by performing a real time estimation of the OPD based on the data continuously recorded. The correction is sent to the VLTI delay lines at a rate of about $1 \mathrm{~Hz}$. Finally, the photometric data are recorded in two more files.

In the first file, only one shutter is opened, corresponding to the calibration of the flux from the first telescope, here UT1, and the flux is then divided by the MIDI beam splitter and falls on two different regions of the detector. The total flux is determined separately by chopping between the object and an empty region of the sky, then the source flux is computed by subtraction. Once it has been done, the same procedure is carried out again for the beam arriving from UT3.

We performed the spectral reduction and fringe calibration using a software developed at the Max-Planck Institut für Astronomie in Heidelberg, written in $\mathrm{IDL}^{1}$. The reader is referred to Leinert et al. (2004) for an extensive discussion on MIDI data reduction and error handling.

The photometric datasets used for the calibration of the contrast of the dispersed fringes are also used for the spectrum flux calibration. The frames obtained on the target and the ones obtained on the sky are averaged. Then, the averaged sky frame is subtracted from the averaged target frame. In the resulting integration, the spectral axis is oriented along the horizontal detector axis. For each detector column, a Gaussian function is fitted to the peak. The position of the spectrum in all illuminated columns, as a function of the column number, is fitted by a quadratic polynomial. In a similar way, the width of the spectrum is fitted by a linear function. This procedure is carried out on both photometric datasets, corresponding to telescopes UT1 and UT3, respectively. Both fits are then averaged and used to create a mask consisting of a Gaussian function with the average position and width of the spectra for each column. Finally, a weighting function is computed to create a mask for the extraction.

The photometric masks are also used to extract the fringe information. Each frame of the fringe data is reduced to a onedimensional spectrum by multiplying it by the mask, integrating in the direction perpendicular to the spectral dispersion and finally subtracting the two output channels of the beam combiner from each other. The spectra from each scan with the piezo-mounted mirrors are collected and Fourier-transformed from optical path difference (OPD) to frequency space. The fringe amplitude at each wavelength is then obtained from the power spectrum. We typically co-added the signal by bins of four pixels in the dispersion direction to improve the signal-tonoise ratio.

The scans where fringes were actually detected are selected based on the white-light fringe amplitude, i.e. the fringe amplitude that is seen after integrating over all usable wavelengths.

\footnotetext{
1 Package available at this address: http://wWw .mpia-hd.mpg.de/MIDISOFT/
}

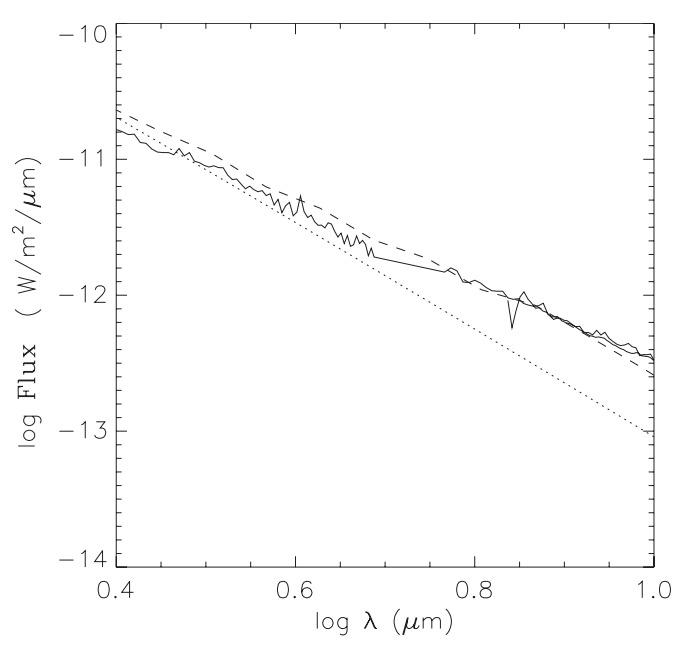

Fig. 1. alpha Ara flux measured by the VLTI/MIDI $(8-13 \mu \mathrm{m})$ instrument with ISO data $(2.5-11.5 \mu \mathrm{m})$ plotted as solid line compared with the theoretical SED of the star alone (dashed line) and the star+envelope emission (dotted line) assuming a distance of $105 \mathrm{pc}$. Note the IR excess starting around $5 \mu \mathrm{m}$, i.e. $\log \lambda=0.7$.

Table 2. Calibrator star parameters, from the MIDI calibrator catalogue (Van Boekel et al. 2005).

\begin{tabular}{lccc}
\hline \hline Calibrator & $\begin{array}{c}\text { Spectral } \\
\text { type }\end{array}$ & $\begin{array}{r}\text { Flux }^{i} \\
(\mathrm{Jy})\end{array}$ & $\begin{array}{c}\text { Uniform disk } \\
\text { diameter (mas) }\end{array}$ \\
\hline HD 139 997 & K5 III & 14.5 & $3.46 \pm 0.38$ \\
HD 152 786 & K3 III & 82.2 & $7.21 \pm 0.21$ \\
HD 165 135 & K0 III & 16.3 & $3.33 \pm 0.05$ \\
HD 168 454 & K3 IIIa & 43.7 & $5.78 \pm 0.15$ \\
\hline
\end{tabular}

${ }^{i}$ IRAS $12.5 \mu$ m flux.

The histogram of all white-light fringe amplitudes within a fringe track dataset usually shows a small peak near zero, and a broad peak at higher amplitudes. We interactively set a threshold just below this broad peak, and average the fringe amplitudes of all scans with a white-light fringe amplitude higher than this threshold to give the final fringe amplitude as a function of wavelength. The raw visibility is obtained by dividing the fringe amplitude by the total flux. The calibrated visibility is obtained by dividing the raw visibility of an object by that of a calibrator. The photometrically calibrated flux creating the fringes is called correlated flux.

The interferometric and photometric data are presented in Table 1 and the parameters of the interferometric calibrators are given in Table 2 .

The flux calibration has been performed with the data of June 16 only. Alpha Aql was considered as a good calibrator for the absolute flux extraction and we have used the ISO observations of this star as a flux reference (but has not been used as an interferometric calibrator). An independent calibration is performed for the individual spectra from each part of the detector and each telescopes. The airmass is also corrected independently for each of them by using the observations of one calibrator for two different times. Figure 1 shows the VLTI/MIDI Spectral Energy Distribution (SED) with the ISO data and the 
theoretical SED obtained with the SIMECA code (see hereafter).

The errors on the observed visibilities are mostly systematic. The statistical signal-to-noise ratio on the white light fringe amplitudes is 5-10 at minimum and much better after adding the several hundred scans taken per interferometric measurement. Most of the time, the dominant error source is the fluctuation of the photometry which occur between the fringe recording and the photometry recording from the individual telescopes which are separated in time by 2 to $10 \mathrm{~min}$. This affects the fringe signal for object and calibrator measurements. Comparing the raw visibilities observed for different calibrator stars over one night, the standard deviation of these values under good conditions amounts to $\pm 5-10 \%$ (relative) at the red and blue end of the spectrum while for adverse conditions these numbers have to be multiplied by a factor of 2-2.5. These fluctuations are much larger for instance than the systematic error expected from the diameters of the calibrators and are mostly achromatic (Leinert et al. 2004). Yet, the instrumental visibility curve of MIDI is very stable in shape, i.e. the slope of the curve typically varies by less than $3-5 \%$. The visibilities observed on calibrators ("instrumental visibility") rises from about 0.4 at $8 \mu \mathrm{m}$ to about 0.7 at $13 \mu \mathrm{m}$, rather repeatable from night to night (i.e. it means that, in good atmospheric conditions, the absolute level is dependant on the photometric variations and hence the stability of the atmospheric conditions but the shape of the visibility curves is mostly an instrumental parameter only weakly affected by the atmosphere).

The data reduction described previously does not consider the variation of the photometry during the 1-2 min needed to record the data and the visibility estimation is usually based on the mean photometric flux. Moreover, under good atmospheric conditions, the statistics of the correlated flux per individual scan follow roughly a Gaussian curve and the mean of this correlated flux is used for the visibility estimation. The main origin of the variability of the correlated flux is the high frequency photometric fluctuations, the atmospheric piston and the quality of the fringe tracking (i.e. the instrumental piston).

During the night of June 17, 2003 thin cirrus clouds passed through the telescope beams. The seeing was quite stable, below $0.5^{\prime \prime}$ during all the night but the standard deviation of the flux from the target pointed by the (visible) seeing monitor ${ }^{2}$ oscillated between 0.02 and 0.05 between $3 \mathrm{~h}$ and $8 \mathrm{~h}$ (UT time), indicating the passage of thin cirrus clouds. These fluctuations seem correlated with an acceleration of the atmosphere turbulence with a coherence time in visible wavelengths decreasing from $\tau_{0}=5.5 \mathrm{~ms}$ at $3 \mathrm{~h}$ UT to $\tau_{0}=2 \mathrm{~ms}$ at $7 \mathrm{~h} 30 \mathrm{UT}$. By comparison, the photometric fluctuations of the seeing monitor calibrator on the 16th of June are below 0.01 during the entire night although the turbulence was also quite rapid with $\tau_{0}=3 \mathrm{~ms}$. The variability of the atmosphere during the June, 17 night was such that a careful inspection of the stability of the photometry and the correlated flux recorded was necessary. The passages of the thin cirrus were easily recognized by a rapid drop

\footnotetext{
2 This information has been extracted from the ESO Ambient conditions database of Paranal observatory: http://archive.eso.org/
}

of the flux lasting between 0.5 and $2 \mathrm{~s}$ during the photometric record and by a simultaneous photometric and correlated flux drop in the tracking files. The frames and the scans concerned were excluded from the data reduction process: this represent about $60 \%$ of the photometry and $50 \%$ of the scans, i.e. about 800 frames were left from each photometric files and about 100 scans were used for the visibility estimation. Of course, this treatment, applied to alpha Ara and its calibrators, is a first order correction and the standard deviation of the instrumental visibility curves of the calibrators observed between $2 \mathrm{~h}$ UT and 9h UT is still large compared to a good observing night, of the order of $18 \%$ whereas the standard deviation of the previous night is only $8 \%$. Fortunately, the longest baseline of $102 \mathrm{~m}$ was used during the best night. The error bars reported in Figs. 6 and 7 reflect the standard deviation computed by this mean.

\subsection{Spectroscopic data}

For an investigation of the variability of alpha Ara this study makes use of a set of 42 echelle spectra, taken over 69 nights in May to July 1999. The data were secured with the HEROS instrument, attached to the ESO-50 cm telescope. The resolving power is $\Delta \lambda / \lambda=20000$, recording the regions from 350 to $565 \mathrm{~nm}$ and from 585 to $860 \mathrm{~nm}$ in the blue and red channel simultaneously. The spectra have a typical signal-to-noise ratio of 170 (Fig. 2).

Complementary spectra have also been recorded quasisimultaneously with the VLTI run in order to know whether alpha Ara has shown IR emission lines. We observed alpha Ara in the $J 2$ band $(1.2283-1.2937 \mu \mathrm{m})$ using the $1.6 \mathrm{~m}$ PerkinElmer telescope and Coudé spectrograph (with $R=10000$ ) at the Observatório do Pico dos Dias, Laboratório Nacional de Astrofísica (LNA), Itajubá, Brasil. In total, five stellar spectra were recorded with the Câmara Infravermelho (CamIV) detector, August 13, 2003. Each spectrum corresponds to a different position of the star along the spatial axis of the entrance slit, allowing us to use the median of the five twodimensional frames, from which the average bias frame was subtracted, as a sky background. After dividing the stellar and sky background frames by the average flat field, the sky was subtracted from two-dimensional stellar spectra. The extraction of one-dimensional spectra, as well as wavelength calibration, using an Ar-Ne calibration lamp, was performed with standard IRAF ${ }^{3}$ packages. Finally, the continuum was normalized to unity.

The observed $\mathrm{Pa} \beta$ line profile is shown in Fig. 3. The emission intensity was $I / I_{\mathrm{c}} \approx 2.3$, while the violet-to-red peak height ratio $V / R$ was 1.17 . Even if the spectroscopic data were not recorded fully simultaneously with the interferometric ones, available observations of typical timescales of the spectral changes of alpha Ara, such as the above-described HERos data, justify the assumption that the circumstellar environment of alpha Ara has not changed significantly between June and

\footnotetext{
${ }^{3}$ IRAF is distributed by the National Optical Astronomy Observatories, which is operated by the Association of Universities for Research in Astronomy (AURA), Inc., under cooperative agreement with the National Science Foundation.
} 

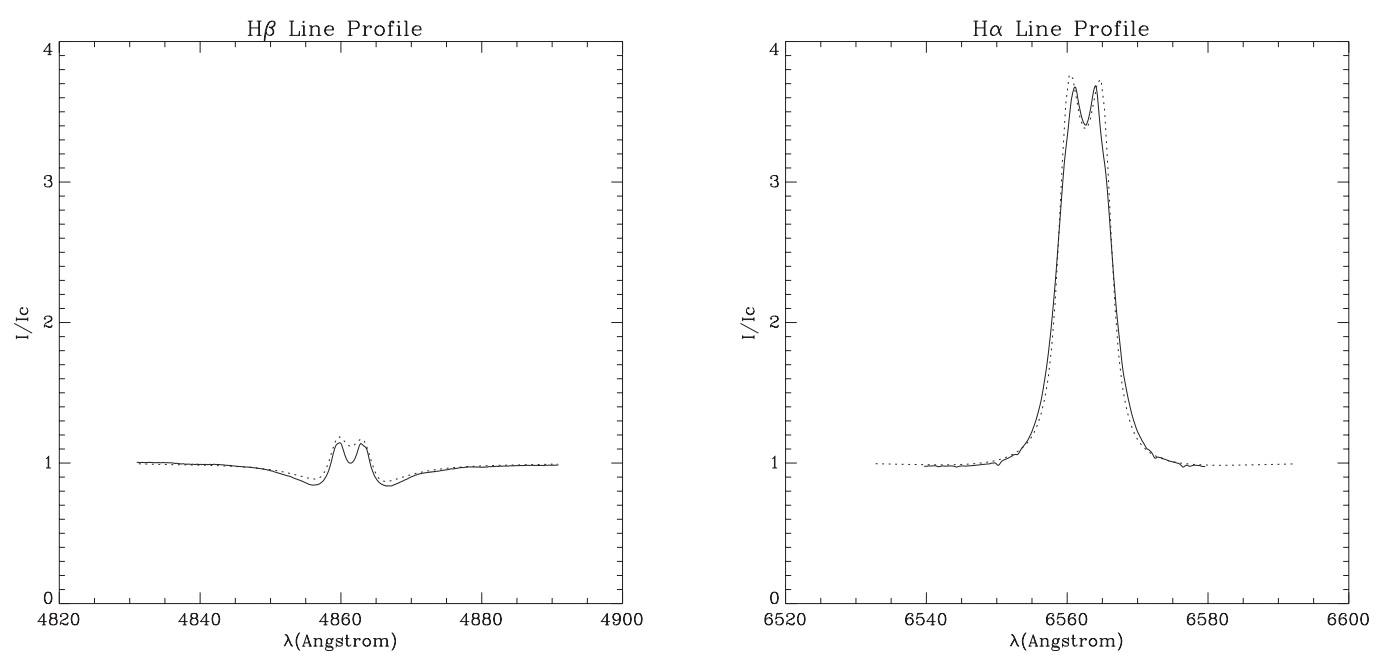

Fig. 2. $\mathrm{H} \alpha$ and $\mathrm{H} \beta$ line profiles observed in mid-1999 (solid lines) compared with the best SIMECA fits (dotted lines).
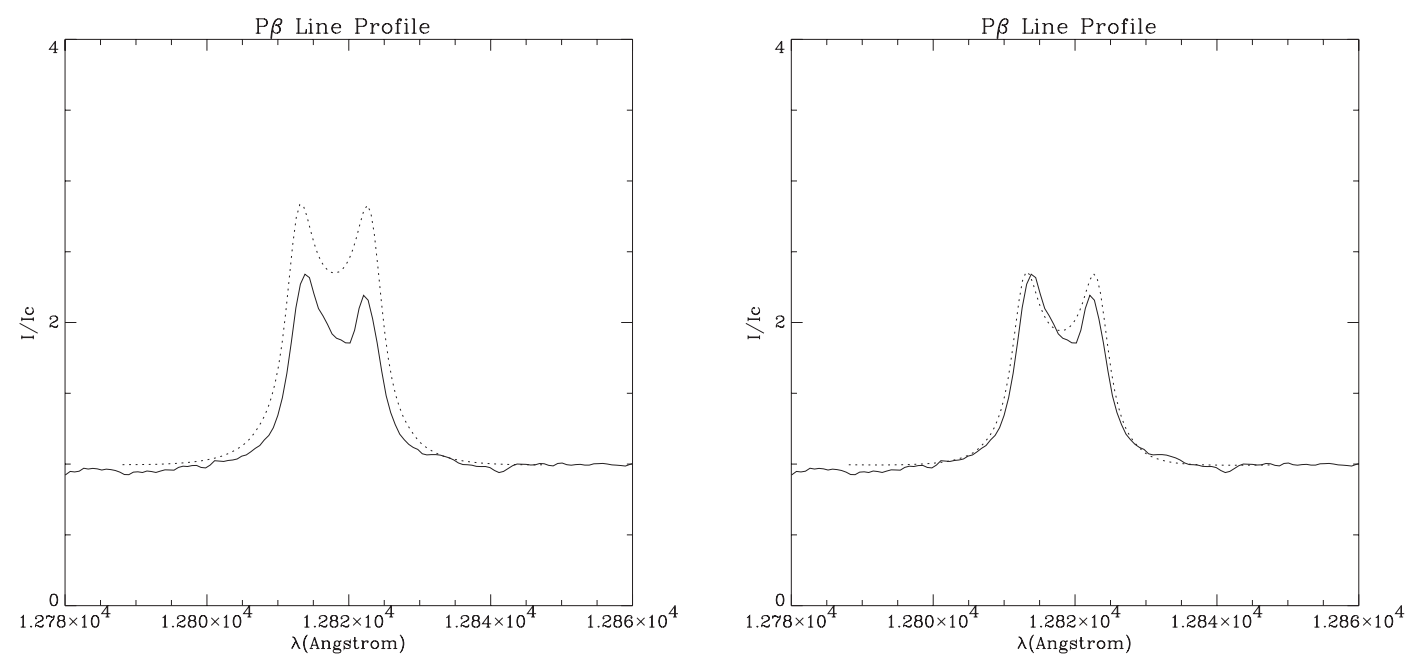

Fig. 3. a) Observed $\mathrm{Pa} \beta$ line profile (mid-2003, solid line) compared to the modeled one based on the parameters determined by the fit of the $1999 \mathrm{H} \alpha$ and $\mathrm{H} \beta$ line profiles (dotted line). b) Observed $\mathrm{Pa} \beta$ line profile (solid line) and the best fit from the SIMECA code (dotted line). To compensate for the effects of non-simultaneous observations, the density of the disk was allowed to vary in a small range. This fit has been obtained with a disk density lower by $25 \%$ compared to the best model based on $1999 \mathrm{H} \alpha$ and $\mathrm{H} \beta$ lines.

August 2003. This $\mathrm{Pa} \beta$ line profile, combined with the interferometric data, constrains the physical parameters of the circumstellar environment of alpha Ara, as it will be shown in the following sections.

\section{The SIMECA code and the envelope parameters used for the modeling of alpha Ara}

In order to constrain the physical parameters of the circumstellar environment of alpha Ara, we have used the SIMECA code. This code, described in previous papers (see Stee \& Araùjo 1994; Stee et al. 1995; Stee \& Bittar 2001), has been developed to model the environment of active hot stars. SIMECA computes line profiles, Spectral Energy Distributions (SEDs) and intensity maps, which can directly be compared to high angular resolution observations. The envelope is supposed to be axi-symmetric with respect to the rotational axis. No meridional circulation is allowed. We also assume that the physics of the polar regions is well represented by a CAK type stellar wind model (Castor et al. 1975) and the solutions for all stellar latitudes are obtained by introducing a parameterized model which is constrained by the spectroscopic and interferometric data. The inner equatorial region is dominated by rotation, therefore being quasi Keplerian. The ionization-excitation equations are solved for an envelope modeled in a $410 * 90 * 71$ cube. In order to reproduce the $\mathrm{Pa} \beta$ profile we have computed the line corresponding to the transitions between the 5-3 atomic levels. The populations of the atomic levels are strongly altered from their Local Thermodynamic Equilibrium (LTE) values by nonLTE conditions in the wind. For computation, we start with the LTE populations for each level, and then compute the escape probability of each transition in the wind, obtaining up-dated populations. By using these populations as input values for the next step, we iterate until convergence. The basic equations of the SIMECA code are given in detail by Stee et al. (1995).

To take into account the photospheric absorption line, we assume the underlying star to be a normal B3 Ve star with $T_{\text {eff }}=18000 \mathrm{~K}$ and $R=4.8 R_{\odot}$ and synthesize the 
Table 3. Stellar parameters used for the modelling of alpha Ara with SIMECA.

\begin{tabular}{cc}
\hline \hline Spectral type & $\mathrm{B} 3 \mathrm{Ve}$ \\
$T_{\text {eff }}$ & $18000 \mathrm{~K}$ \\
Mass & $9.6 M_{\odot}$ \\
Radius & $4.8 R_{\odot}$ \\
Luminosity & $2.2 \times 10^{3} L_{\odot}$ \\
\hline
\end{tabular}

photospheric line profiles using the SYNSPEC code by Hubeny (Hubeny 1988; Hubeny \& Lanz 1995). The resulting line profile is broadened by solid-body rotation and might be further altered by absorption in the part of the envelope in the line of sight towards the stellar disk.

The stellar parameters (Table 3) are important mainly to define the distance and the luminosity of the source (Fig. 1) and represent the first step of the iterative process leading to a model of the envelope as described below. In the scope of this model, we have computed the $\mathrm{H} \alpha, \mathrm{H} \beta$ (Fig. 2) and $\mathrm{Pa} \beta$ (Fig. 3) line profiles.

The critical constraint of this study is based on the fact that the $\mathrm{H} \alpha$ and $\mathrm{H} \beta$ lines (Fig. 2), as well as the the $\mathrm{Pa} \beta$ line (Fig. 3) are strongly in emission, whereas from Fig. 6 the visibilities are clearly around unity, i.e. the envelope in the $N$ band is nearly unresolved. Hence, the circumstellar density must be large enough to produce Balmer lines in emission while at the same time this density must be low enough and/or the circumstellar environment must be well delimited to keep the envelope unresolved. The emissive lines of many Be stars like alpha Ara exhibit a characteristic double peaked shape and the "violet" and "red" peaks are denoted $V$ and $R$. This shape provides stringent constraints on the wind parameters and particularly on the inclination of the system.

In order to obtain matching parameters derived from both line profiles and interferometric measurements we have adopted the following strategy:

1. the density $\rho_{0}$ at the base of the wind is fixed, having the greatest influence on the emission line intensity;

2. a range of inclination angles is chosen in order to match the $V$ and $R$ peak separation, $V$ and $R$ peak intensities and line wings with the observed profile. In this case, the range is 40-50 ;

3 . in the next step, the $m 1$ and $m 2$ parameters are varied, where $m 1$ describes the variation of the mass flux from the pole to the equator according to:

$\phi(\theta)=\phi_{\text {pole }}+\left[\left(\phi_{\text {eq. }}-\phi_{\text {pole }}\right) \sin ^{m 1}(\theta)\right]$

where $\theta$ is the stellar colatitude. The parameter $m 2$ describes the variation of the terminal velocity $v_{\infty}$ as a function of the stellar latitude:

$v_{\infty}(\theta)=V_{\infty}($ pole $)+\left[v_{\infty}(\right.$ eq. $)-v_{\infty}($ pole $\left.)\right] \sin ^{m 2}(\theta)$

where $V_{\infty}$ (pole) and $V_{\infty}$ (eq) are respectively the polar and equatorial terminal velocities;

4. the ratio between the equatorial and polar mass flux:

$$
C 1=\frac{\Phi_{\mathrm{eq}}}{\Phi_{\mathrm{pole}}}
$$

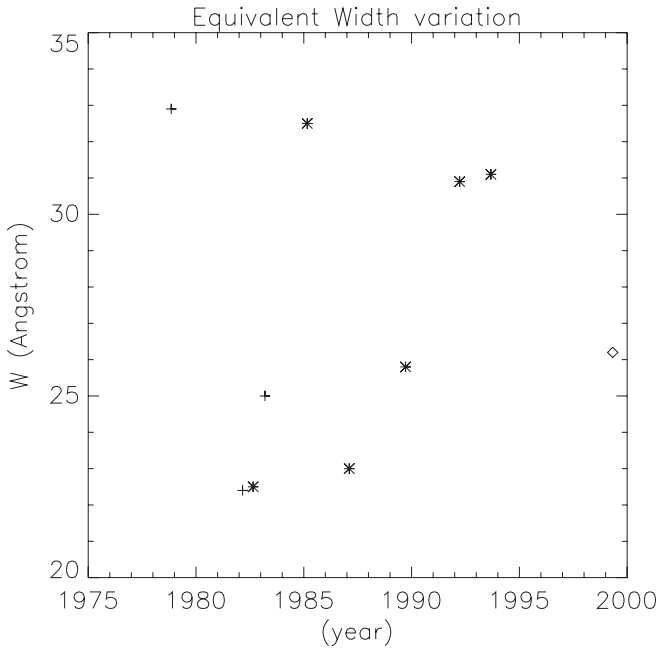

Fig. 4. Variation of the $\mathrm{H} \alpha$ equivalent width $(E W)$ with time. The data were taken from Dachs et al. (1990): crosses; Hanuschik et al. (1995): stars and this work: diamond.

is typically between $10^{1}$ and $10^{4}$ (Lamers \& Waters 1987), having a large influence on the wings of the line profile;

5. following the above procedure, the $\mathrm{H} \alpha$ and $\mathrm{H} \beta$ line profiles are fitted simultaneously to obtain the general envelope parameters;

6. with the parameters reproducing well the 1999 appearance of $\mathrm{H} \alpha$ and $\mathrm{H} \beta$, the modelled $\mathrm{Pa} \beta$ line profile has a greater intensity than actually observed in 2003 (Fig. 3). In the following, we assumed that the only parameter that might have changed between 1999 and 2003 is the density at the base of the envelope. In fact, with a base density decreased by about $25 \%$, a better agreement between the observed and modeled $\mathrm{Pa} \beta$ profiles is obtained (Fig. 3);

7. the comparison between the theoretical SED we obtained and the MIDI and ISO data (Fig. 1) is used to constrain the distance of alpha Ara. The adopted a distance is based on the hypothesis on the star parameters and the fitted parameters of the circumstellar environment of alpha Ara which settle the infrared excess and the visual reddening (see discussion Sect. 5.2);

8. in a final step, we compute the intensity maps in spectral channels of $0.2 \mu \mathrm{m}$. Using the distance estimated in the previous step, we obtain theoretical visibilities which are compared to the VLTI/MIDI data. We found that the critical parameters to fit the observed visibilities are the distance to the star, the inclination angle, the $m 1$ value and the density at the base of the wind $\rho_{0}$.

During the above described procedure, more than 500 models were computed using the SIVAM II Alpha ES45 EV68 workstation, build on 3 servers with each of four $1 \mathrm{GHz}$ CPU and 24 GB of RAM at the Observatoire de la Côte d'Azur. To compute one model it takes about 20 min of CPU time.

The variability of the circumstellar environment of $\alpha$ Ara is traced by the variations of the $\mathrm{H} \alpha$ line equivalent width $(E W)$ reported in the literature by Dachs et al. (1990), Hanuschik et al. (1995) and this work. From Fig. 4 it is evident that we 
Table 4. Best model parameters for the alpha Ara circumstellar environment obtained by fitting the visibilities and the 2003 Pa $\beta$ line profile with SIMECA.

\begin{tabular}{lcc}
\hline \hline Parameter/result & Value & Uncertainty \\
\hline Distance & $105 \mathrm{pc}$ & $10 \mathrm{pc}$ \\
Inclination angle $i$ & 45 & 5 \\
Photospheric density $\left(\rho_{\text {phot }}\right)$ & $1.2 \times 10^{-12} \mathrm{~g} \mathrm{~cm}^{-3}$ & linked to C1 \\
Photospheric expansion velocity & $0.07 \mathrm{~km} \mathrm{~s}^{-1}$ & 0.01 \\
Equatorial rotation velocity & $300 \mathrm{~km} \mathrm{~s}^{-1}$ & 20 \\
Equatorial terminal velocity & $170 \mathrm{~km} \mathrm{~s}^{-1}$ & 20 \\
Polar terminal velocity & $2000 \mathrm{~km} \mathrm{~s}^{-1}$ & 500 \\
Polar mass flux & $1.7 \times 10^{-9} M_{\odot} \mathrm{year}^{-1} \mathrm{sr}^{-1}$ & $0.5 \times 10^{-9}$ \\
$m 1$ & 0.3 & 0.1 \\
$m 2$ & 0.45 & 0.05 \\
$C 1$ & 30 & linked to $\rho_{\text {phot }}$ \\
Mass of the disk & $2.3 \times 10^{-10} M_{\odot}$ & - \\
Mass loss & $6.0 \times 10^{-7} M_{\odot}$ year $^{-1}$ & - \\
\hline
\end{tabular}

cannot straightforwardly use parameters derived from the $1999 \mathrm{H} \alpha$ and $\mathrm{H} \beta$ line profiles to model the $\mathrm{Pa} \beta$ line profile observed in 2003 , i.e. close to our interferometric run. It is most likely that some physical parameters have changed between these two epochs.

\section{Results}

\subsection{Theoretical line profiles and SED}

The $\mathrm{H} \alpha$ line observed 1999 shows strong emission of $I / I_{\mathrm{c}} \approx 3.7$, which is well reproduced by SIMECA with the stellar parameters given in Table 3 and the envelope parameters from Table 4 (Fig. 2).

With a wind base density decreased by $25 \%$, producing a good agreement for the $\mathrm{Pa} \beta$ line profile (Fig. 3), the model gives an $\mathrm{H} \alpha$ line profile with a $I / I_{\mathrm{c}}$ ratio $\approx 3$ (Fig. 5). Unfortunately, we do not have $\mathrm{H} \alpha$ line profiles taken simultaneously with the VLTI observations that could confirm such a decrease.

Even though Fig. 3 shows that the $\mathrm{Pa} \beta$ line profile modelled with SIMECA is in good agreement with the observed one, the modelled $V / R$ is less than unity, whereas the observed one is $V / R \approx 1.17$. SIMECA is based on a radiative wind model for which the gas is outflowing from the star with a polar and equatorial velocity of respectively 2000 and $170 \mathrm{~km} \mathrm{~s}^{-1}$. In order to obtain a $V / R>1$, it would be necessary to introduce an asymmetry like a global one-armed oscillation, as seen by Berio et al. (1999) and Vakili et al. (1998) for the $\gamma$ Cas and $\zeta$ Tau equatorial disks. Nevertheless, the global agreement of observation and model both in profile shape and intensity indicates that, under the general assumptions of the SIMECA model, the global kinematics and density distribution within the envelope are well reproduced by the assumed model parameters.

The computed SED between 2 and $10 \mu \mathrm{m}$ is shown in Fig. 1, together with the data recorded by VLTI/MIDI $(8-13.5 \mu \mathrm{m})$ and the ISO satellite $(2.5-11 \mu \mathrm{m})$. The observed SED and the modelled curve match for a source distance

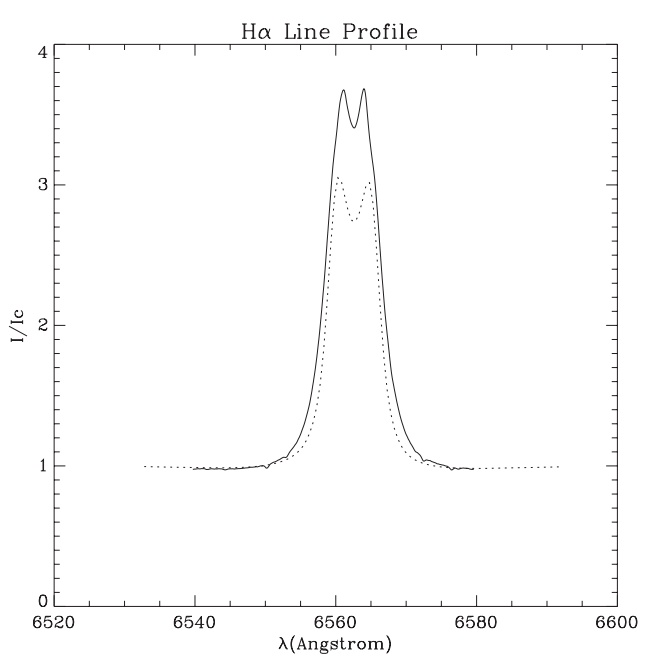

Fig. 5. H $\alpha$ line profile obtained in April 1999 with the HEROS instrument at la Silla (Chile) and the SIMECA fit (dotted line) for the base density used to fit the $2003 \mathrm{~Pa} \beta$ profile. The strength of the line is comparable of the one reported in 1982 or 1987, for instance see Fig. 2 by Hanuschik et al. (1995).

of $105 \mathrm{pc}$. This distance is significantly larger than the one obtained from the Hipparcos satellite (see discussion in Sect. 5). In Fig. 1 the continuum emitted by the central star is approximated as a $T_{\text {eff }}=18000 \mathrm{~K}$ black body radiation plotted as a dashed line. The total, i.e. free-free and free-bound emission from envelope + star, is indicated by the dotted line. Clearly, the infrared excess produced by the circumstellar envelope is mandatory in order to fit the observed data (solid line).

\subsection{Theoretical visibilities}

The parameters obtained by the above modeling of the emission line profiles, and the density required to fit the $2003 \mathrm{~Pa} \beta$ line, can now be used to compute the expected visibility curves for a distance of $105 \mathrm{pc}$, as estimated from the fit of the SED. The resulting visibility curves are plotted in Fig. 6, clearly 

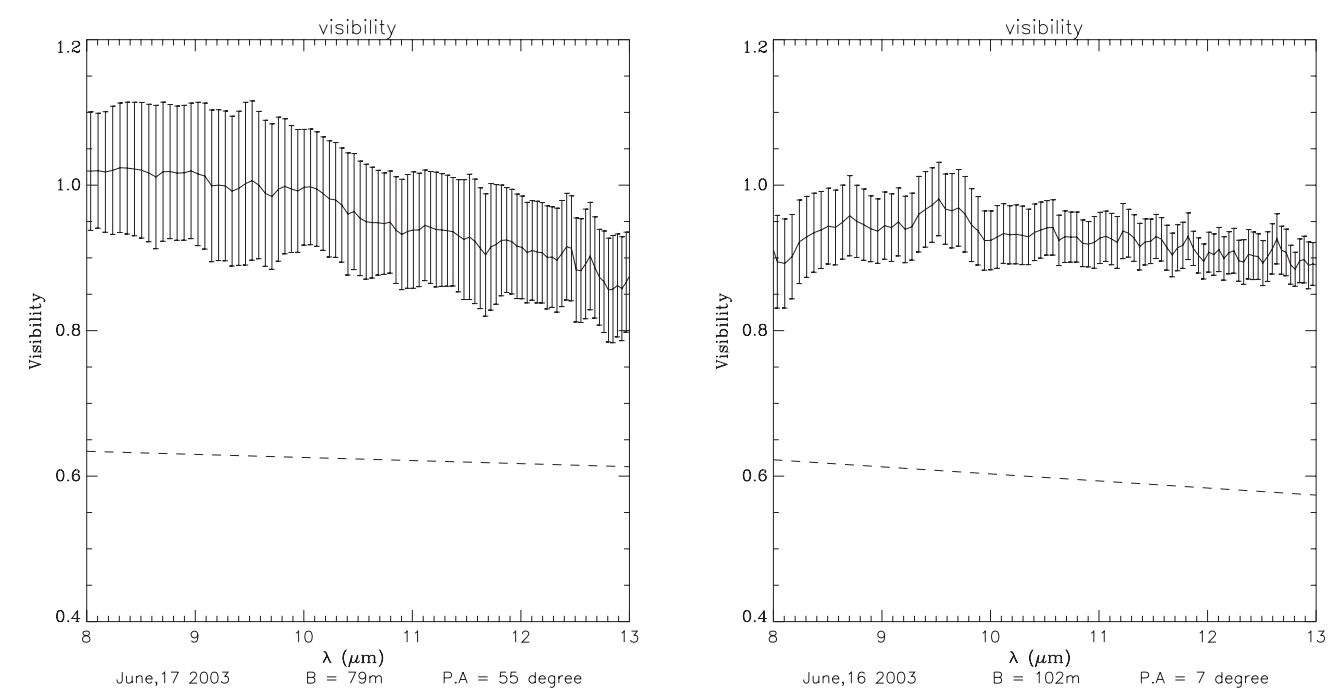

Fig. 6. VLTI/MIDI data for the $79 \mathrm{~m}$ (left) and $102 \mathrm{~m}$ (right) baselines and theoretical visibilities from SIMECA (dashed line) for the distance of 105 pc estimated from our SED fit (see Fig. 1). The error bars are equivalent to one sigma.
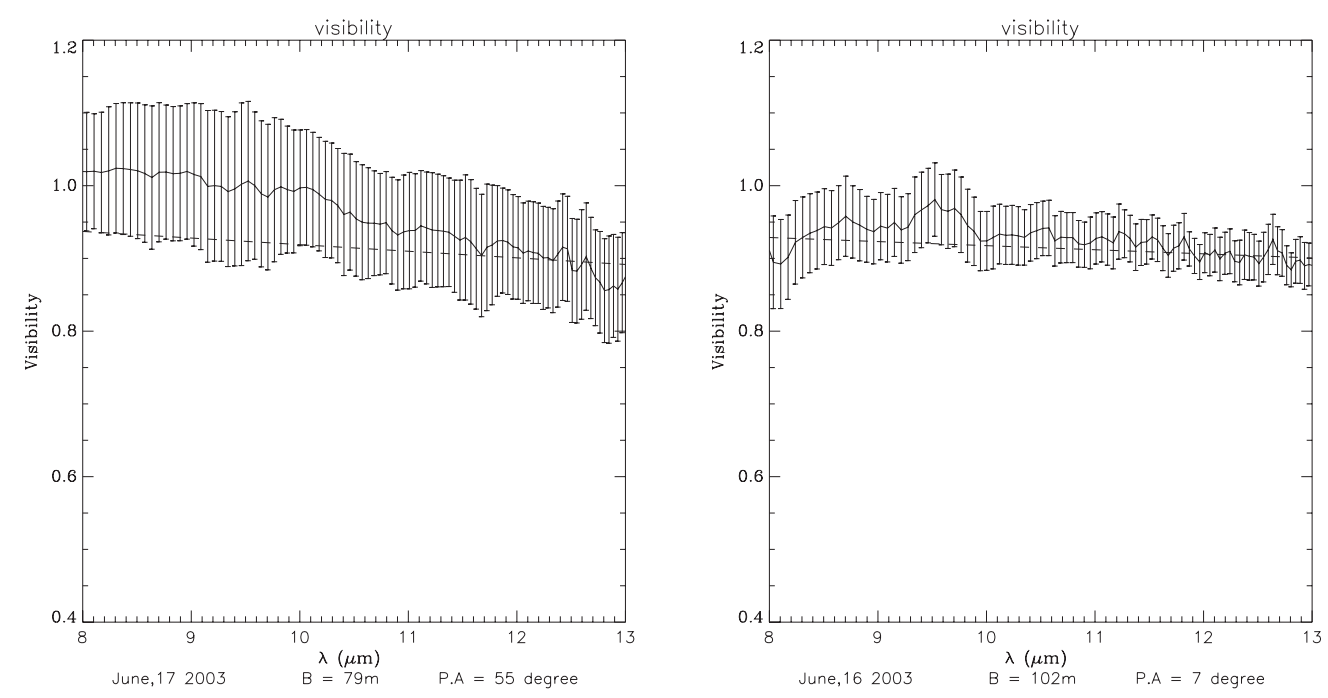

Fig. 7. VLTI/MIDI data for the $79 \mathrm{~m}$ (left) and $102 \mathrm{~m}$ (right) baselines and theoretical visibilities from SIMECA (dashed line) for a truncated disk at $22 R_{\star}$ by a possible companion. The error bars are equivalent to one sigma.

showing that the modeled envelope should be well resolved at $79 \mathrm{~m}$ and $102 \mathrm{~m}$ (mean visibility $V_{\text {mean }} \approx 0.63$ ) whereas the VLTI/MIDI data without any doubt have hardly resolved the target $\left(V_{\text {mean }} \approx 1\right)$. However, the observed visibility curves in Fig. 6 may indicate that the envelope is more resolved for the longer wavelengths. This effect, if true, is more or less reproduced by our model and is more obvious for the $102 \mathrm{~m}$ baseline where the theoretical visibility is 0.62 at $8 \mu \mathrm{m}$ and 0.57 at $13 \mu \mathrm{m}$.

Nevertheless, we did not succeed in obtaining model parameters reproducing both the Balmer lines and the visibility curves at the same time: at a distance of $105 \mathrm{pc}$ the model gives an envelope which is clearly resolved, if the constraint that the $\mathrm{Pa} \beta$ line is in emission with $I / I_{\mathrm{c}} \approx 2.3$ is met. One possibility would be to increase the distance of the star up to $122 \mathrm{pc}$, a distance suggested by Cohen et al. (1998). However, we consider this value as too high in comparison to the Hipparcos distance (see also the discussion in Sect. 5).
The modeled envelope is more or less spherical since the input parameter $m 1=0.3$ corresponds to an opening angle of $160^{\circ}$ (see Stee 2003), producing only a negligible visibility difference for the envelope seen parallel or perpendicular to the polar axis. Nevertheless, this small flattening may produce the small intrinsic linear polarization of $\mathrm{Pl} \approx 0.6 \%$ measured by McLean \& Clarke (1979) and Yudin et al. (1998), who found a polarization angle $\mathrm{PA}=172^{\circ}$. The inner disk orientation is, therefore, expected to be perpendicular to this direction at $82^{\circ}$. This means that the longer baseline was unfortunately oriented at about $70^{\circ}$ from the expected major axis of the disk. The diagram of the alpha Ara circumstellar envelope projected onto the sky plane (with an arbitrary oblateness) and the VLTI baseline positions is given in Fig. 9.

Considering these difficulties, we have computed a model with a truncated disk. The radius where the truncation occurs was set to $22 R_{\star}$, which was derived using a distance of $105 \mathrm{pc}$ and the fit of the $\mathrm{Pa} \beta$ line (Fig. 7). The theoretical visibilities 

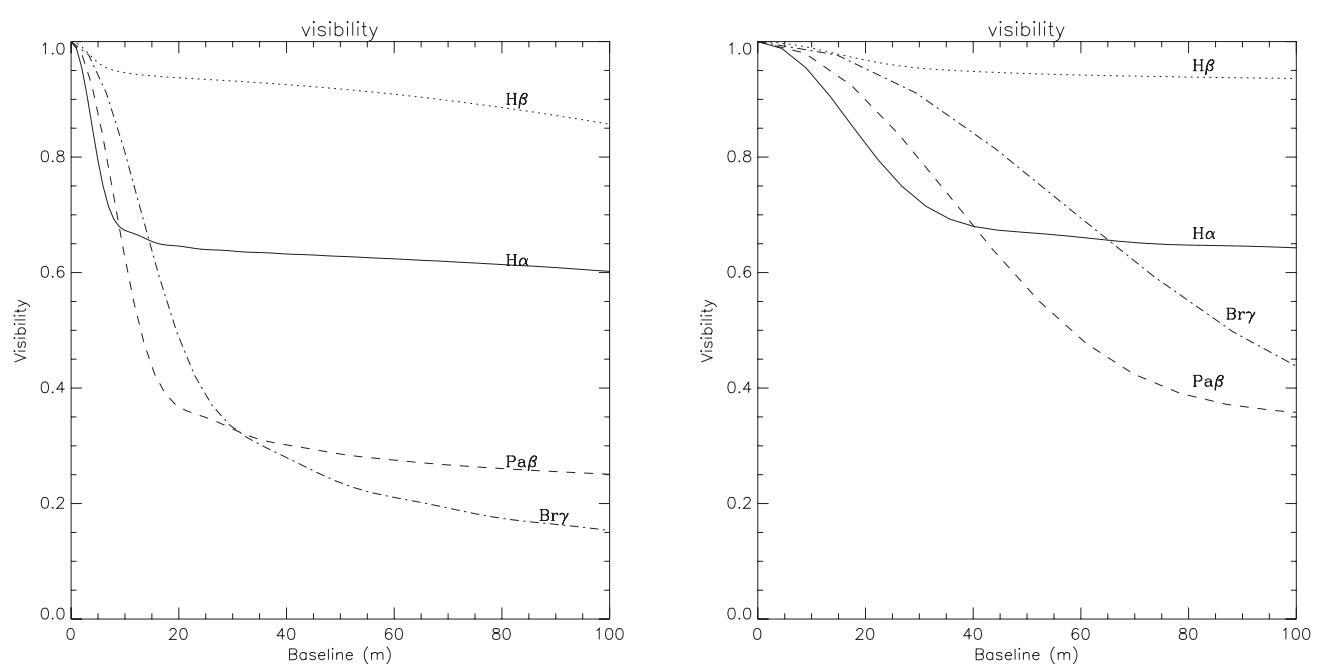

Fig. 8. a) Theoretical visibilities for the $\mathrm{H} \alpha, \mathrm{H} \beta, \mathrm{Pa} \beta$ and $\mathrm{Br} \gamma$ lines for baseline orientations corresponding to the ones observed on June 16, i.e. PA $=7^{\circ}$ onto the sky plane, for both scenarios discussed; b) model data for a disk with the parameters which fits the $2003 \mathrm{~Pa} \beta$ line profile, but not in agreement with the VLTI/MIDI visibilities (Fig. 6). On the right side model data for a disk truncated at $22 R_{\star}$, e.g. by a putative companion, is presented, being in agreement with the observed visibilities (Fig. 7).

in the $\mathrm{H} \alpha, \mathrm{H} \beta, \mathrm{Pa} \beta$ and $\mathrm{Br} \gamma$ lines are plotted in Fig. 8 for the two scenarios. For both models it is clear that at shorter wavelengths, with a $102 \mathrm{~m}$ baseline, the envelope of alpha Ara would be well resolved in the $\operatorname{Pa} \beta$ and $\operatorname{Br} \gamma$ lines $(V \approx 0.2-0.3)$ even for a truncated disk $(V \approx 0.35-0.45)$. In $\mathrm{H} \alpha$ and $\mathrm{H} \beta, V$ is 0.6 and 0.85 , respectively, for the full disk and $V$ is 0.65 and 0.94 for the truncated one. At these wavelengths, the emission comes from the inner part of the disk, which remains unresolved at a distance of $105 \mathrm{pc}$. Consequently, such a truncation of the disk would have only a little impact on the $\mathrm{H} \alpha$ and $\mathrm{H} \beta$ emitting regions, whereas the IR emission, originating from more extended regions, is more affected.

The fact that alpha Ara is nearly unresolved at $8 \mu \mathrm{m}$ with a $102 \mathrm{~m}$ baseline gives an upper limit for the diameter of the emitting envelope of $\phi_{\max }=4 \pm 1.5$ mas, i.e. $14 R_{\star}$ (for $d=74 \mathrm{pc}$ ) or $20 R_{\star}$ (for $d=105 \mathrm{pc}$ ) assuming a uniform disk distribution for the star+disk brightness distribution. If a Gaussian distribution is assumed, the best fit is obtained with a $F W H M=2.5$ mas although the quality if the fit is poorer than for the uniform disk hypothesis. Such a radial extension is in good agreement with the one obtained by Waters et al. (1987) from IRAS Far-IR data, with $\phi_{\text {disk }} \sim 7 R_{\star}$. Indeed, the hypothesis that the star+envelope flux distribution is unchanged from $8 \mu \mathrm{m}$ to $13.5 \mu \mathrm{m}$ is a crude approximation and these estimated extensions must be taken as indicative only. It must be stressed out that the slopes of the visibility curves provided by the model are in good agreement with the data showing that the flux is more concentrated at $8 \mu \mathrm{m}$ than at $13 \mu \mathrm{m}$. Moreover, the angular diameter estimations are mostly based on the visibilities taken with the longest projected baseline at $\mathrm{PA}=7^{\circ}$ to the East, i.e. more or less along the predicted polar direction (see Fig. 9).

In Table 4 we present the results based on the best fit of the alpha Ara August $2003 \mathrm{~Pa} \beta$ line profile and visibilities in the $N$ band.

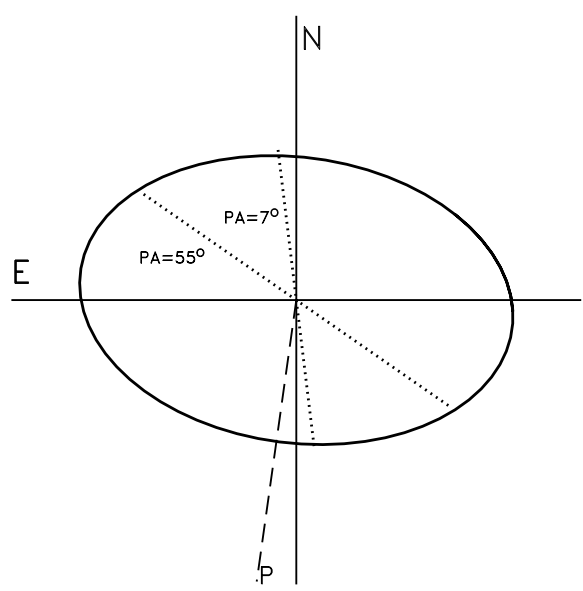

Fig. 9. Diagram of alpha Ara circumstellar envelope projected onto the sky plane (with arbitrary oblateness) based on polarization $(P)$ measurements $\left(\mathrm{PA}=172^{\circ}\right)$ obtained by McLean \& Clarke (1979) and Yudin et al. (1998). The actually observed VLTI baseline positions are shown for June, 16: PA $=7^{\circ}, B=102 \mathrm{~m}$ and June, 17: PA $=55^{\circ}$, $B=79 \mathrm{~m}$.

\section{Discussion}

The characteristics of the disk around alpha Ara seem to have been fundamentally stable over the past century. Only the overall strength of the emission, as traced e.g. by the $\mathrm{H} \alpha E W$ exhibit large variations (see Fig. 4). However, the compilation of spectroscopic observations and the consecutive study of this disk by MIDI have revealed a number of interesting and partly unsuspected characteristics.

\subsection{Variability and suspected binarity}

The Hydrogen recombination line profiles of alpha Ara exhibits (quasi?)-periodic short-term $V / R$ variations with a timescale of 45 days (Mennickent \& Vogt 1991). The timescale for these 
variations measured in the HEROS dataset, however, is better accounted for by a larger period of 70-90 days (see below). Superimposed on these variations are changes in the emission line equivalent widths. For instance, the strength of the $\mathrm{H} \alpha$ emission varies from about 3.2 to more than 4.5 in units of the local continuum in only a few weeks. The period of variations could be the same as the $V / R$ period, but this cannot be shown from our data and it also has not yet been noticed by other investigators. The authors studying the $V / R$ variations concentrated most of their efforts on the shape of the line profile rather than on the absolute strength. Nevertheless, some interesting cases of rapid $V / R$ changes, correlated with strong variations of Balmer line equivalent widths are reported by Mennickent $(1989,1991)$.

Long-term $V / R$ variations are common in Be stars, being attributed to a one-armed oscillation in the Keplerian disk. Rapid $V / R$ variations, however, have not been extensively discussed in the literature. Mennickent (1991) associates fast quasi-periodic variations $(P \leq 100 \mathrm{~d})$ to the smallest disks, i.e. the disks with the lowest equivalent widths in $\mathrm{H} \alpha$. Okazaki (1997) found that for early-type Be stars around B0 radiative effects can explain the confinement of this one-armed oscillations with typical periods of about $10 \mathrm{yr}$. Nevertheless, this model would have difficulties in explaining more rapid $V / R$ variations. Another possibility for such variable asymmetry is the presence of a companion, orbiting at distances larger than the envelope extension. Then, these quasi-periodic line profile variations should be related to the orbital parameters of the putative secondary.

Nevertheless, with respect to the photospheric absorption lines alpha Ara is unusually stable among earlier type Be stars. In a sample of 27 early Be stars observed with HEROS observations, only two stars, including alpha Ara, did not show any detectable line profile variability (Rivinius et al. 2003).

However, this is quite different for the circumstellar emission lines. As well as slow, possibly cyclic long-term changes typical of Be stars, there are changes on a shorter timescale. In particular, the peak-height-ratio of the violet and red peaks of the higher Balmer lines have made one full cycle of low amplitude (about 0.95 to 1.05) during the 69 days of the HERos observations (see Fig. 10). At the same time, the radial velocity of the emission component of the Balmer lines was changing in a cyclic way as well. For instance, Fig. 11 shows this effect for the central absorption of the $\mathrm{H} \beta$-emission, but the same is seen if the radial velocity of the emission peaks is traced, or if other Balmer lines are investigated. Unfortunately, the available data are not good enough to claim such a radial velocity change in the weaker emission lines or in the photospheric absorption lines. The uncertainty of measuring the radial velocity of such lines is much higher than the amplitude seen in Fig. 11. Mennickent et al. (1991) have observed similar changes and gave a cycle length of 0.13 years, or 45 days. However, their Fig. 2 would also support a cycle length of 70 to 80 days, as present in the HEROS data of 1999.

Such behavior is known from a few other Be stars as well. In the cases investigated thoroughly, it was shown to be linked to binarity, although the mechanisms are not yet known (see for instance Koubsky et al. 1997). A search for any spectral

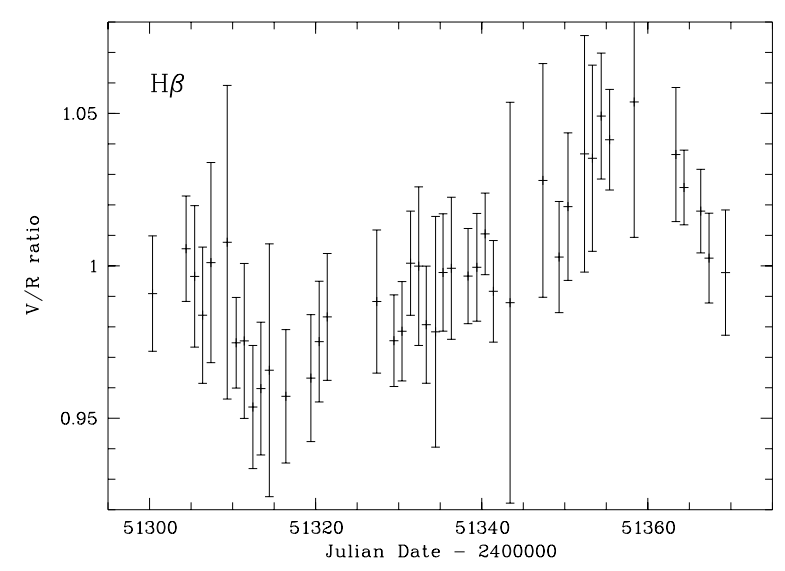

Fig. 10. Variation of the height ratio of violet and red emission peaks of the $\mathrm{H} \beta$ line during the HEROS observations 1999.

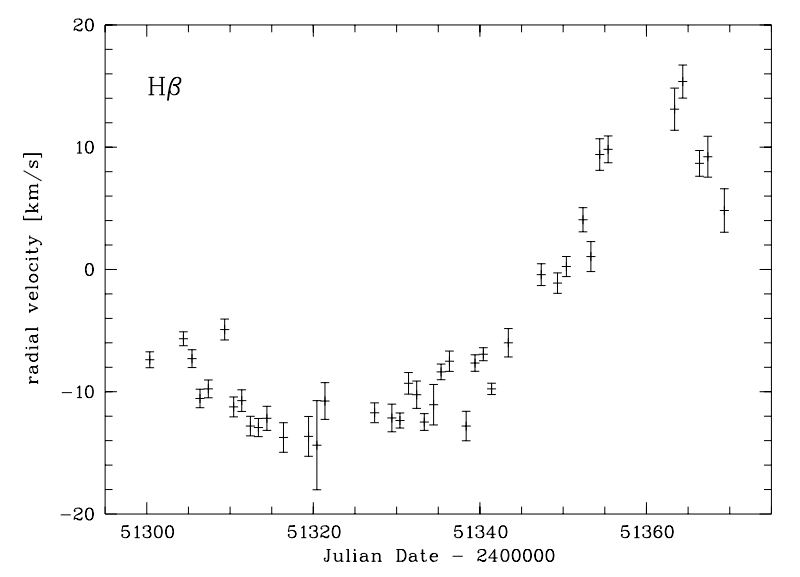

Fig. 11. Radial velocity changes of the central depression of $\mathrm{H} \beta$ during the HEROS observations 1999.

contribution of such a hypothetical companion in the phasebinned HERos spectra of alpha Ara did not return a positive result.

Nevertheless, since a companion as a potential origin for an outer truncation of the disk is important in light of the above results, the properties of such a system are estimated from the following: Using the stellar parameters of Table 3, i.e. a mass of $10 M_{\odot}$, a 70 day period would give a radius of about $154 R_{\odot}$, assuming a circular orbit of a companion with negligible mass. With $R_{\star}=4.8 R_{\odot}$, this corresponds to about 32 stellar radii, which is in agreement with the estimate based on the MIDI/VLTI data for a disk truncated at $25 R_{\star}$, i.e. somewhat smaller than the companion orbit.

Such an orbital dimension can also be estimated by integrating the radial velocity amplitude detected in $\mathrm{H} \beta$ of the order of $12-16 \mathrm{~km} \mathrm{~s}^{-1}$. Assuming a circular orbit, a period of 69 days and testing two inclination angles of $i=30^{\circ}$ and $60^{\circ}$, the mass of the companion would range from $1.4 M_{\odot}(\mathrm{F} 2-4 \mathrm{~V})$ and $2.8 M_{\odot}(\mathrm{A} 2-4 \mathrm{~V})$. The corresponding Roche lobe radii $R_{\text {Roche }}$ are roughly between 15 and $20 R_{\star}$ although we point out that the radial velocity curve shown in Fig. 11 would suggest an eccentricity of about $0.2-0.3$, affecting the previous estimation. 
However, the radial velocities derived from emission lines cannot be taken at face value. Depending on the systemic properties, the radial velocities measured in the central inversion of the circumstellar emission follow the actual radial velocity curves of the stars only loosely. The above orbit size and period, again for the circular case and negligible mass of the companion, result in an orbital velocity of about $18 \mathrm{~km} \mathrm{~s}^{-1}$, which is in the same order of magnitude as the amplitude measured in the emission lines. In some well-investigated binaries like $59 \mathrm{Cyg}$ (Harmanec et al. 2002; Rivinius \& Štefl 2000, $P \sim 29 \mathrm{~d}$ ) or $\phi$ Per (Hummel \& Štefl 2001; Štefl et al. 2000, $P \sim 127$ d), the emission of the Balmer lines is indeed in phase with the orbital motion.

Until now, no evidence has been reported for a possible companion around alpha Ara, but it should be kept in mind that it is difficult to search for low mass companions around bright stars, such as Be stars (see for instance the case of $\gamma$ Cas, Harmanec et al. 2000; Miroshnichenko et al. 2002, period $P \sim 204 \mathrm{~d}$ ). The X-ray flux from alpha Ara is not peculiar and is similar to that of normal B stars $\left(L_{\mathrm{X}}=3.75 \times 10^{28} \mathrm{erg} \mathrm{s}^{-1}\right.$, assuming $d=122 \mathrm{pc}^{4}$, Cohen et al. 1998). The proposed truncation may also help to explain the stringent non-detection of radio emission from alpha Ara at a level of $0.1 \mathrm{mJy}$ at $3.5 \mathrm{~cm}$ and $6.3 \mathrm{~cm}$ (Steele et al. 1998).

Using the NPOI interferometer, Tycner et al. (2004) have recently studied the disk geometry of the Be star $\zeta$ Tau, which is also a well-investigated spectroscopic binary $(P \sim 133 \mathrm{~d}, K \sim$ $\left.10 \mathrm{~km} \mathrm{~s}^{-1}\right)$. They measured the disk extension quite accurately to be well within the Roche radius. This suggests also that this disk may be truncated.

\subsection{Stellar parameters and distance estimation}

The distance used to model the star deviate from the Hipparcos distance by several sigma. The error bar of the Hipparcos measurement is $6 \mathrm{pc}$, and even taking into account a putative correlated error of the order of $1 \mathrm{mas}^{5}$ (Narayanan \& Gould 1999; Pan et al. 2004), the largest distance within 1 sigma could be $87 \mathrm{pc}$. The distance of $105 \mathrm{pc}$ comes straightforwardly from the chosen stellar parameters, i.e. the absolute magnitude as estimated from effective temperature and radius. We notice for instance that Fabregat \& Reglero (1990) obtain a distance of $98 \mathrm{pc}$ from direct $u b v y$ photometry. Zorec $\&$ Briot (1991) obtain $85 \mathrm{pc}$, and note that their estimates of Be star distances seem systematically lower by $20 \mathrm{pc}$ than normal B stars observed in clusters, suggesting that their distance is a lower limit. Also comparing the flux-calibrated IUE archival spectra to Kurucz ATLAS9-data gives a distance of about 105 pc (see Fig. 12), assuming $R_{\star}=4.8 R_{\odot}$ and neglectable extinction in the UV. The latter is probably justified since there is no interstellar extinction towards such a close star

\footnotetext{
${ }^{4}$ This implies a luminosity $L_{\mathrm{X}}=1.02 \times 10^{29} \mathrm{erg} \mathrm{s}^{-1}$, assuming the Hipparcos distance $d=74 \mathrm{pc}$, which is still in the range of luminosities encountered for this spectral type by Cohen et al. (1998).

5 This correlated error is realistic since Narayanan \& Gould (1999) report spatially correlated errors of Hipparcos up to 2 mas in the direction of the Pleiades and Hyades clusters.
}

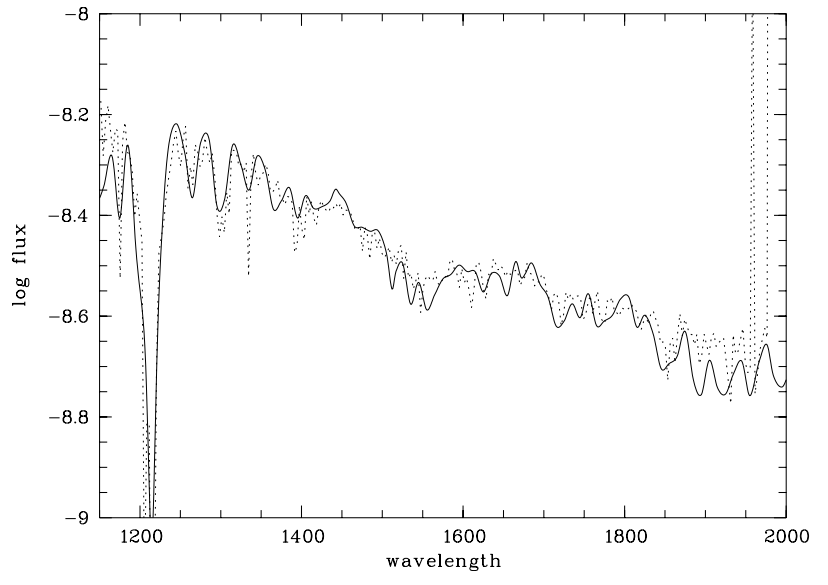

Fig. 12. Average of the IUE-spectra of alpha Ara (dotted) compared to a theoretical spectrum with $T_{\text {eff }}=18000 \mathrm{~K}$ and $\log g=4.0$ (solid line).

(Dachs et al. 1988), and the circumstellar effects for a non-shell star (see discussion below) should not affect the UV-regime. We note also that the parameters of alpha Ara and its hydrogen environment provided by the SED fits of Dachs et al. (1988) are close to the ones presented in this paper, in particular the estimated circumstellar reddening.

The available spectral classifications of the star are all around B3 Ve, so that it can be assumed that the effective temperature is well constrained at about $18000 \mathrm{~K}$. Instead, the main source of uncertainty in the model may be the radius, which arbitrarily has been chosen to be $4.8 R_{\odot}$, based on the statistical value given by Tokunaga in Allen's "Astrophysical Quantities" (2000).

However, alpha Ara has a high $v \sin i$ of about $270 \mathrm{~km} \mathrm{~s}^{-1}$ (Dachs et al. 1990; Yudin 2001; Chauville et al. 2001). With such a large projected rotational velocity, the hypothesis of a uniform disk radius is questionable and the radius used is most probably underestimated as demonstrated by the recent observations of alpha Eri (Achernar et al. 2003). This implies an increase of the illuminating area and strengthens the difficulty of putting the star at the Hipparcos distance. Without considering any reddening, and keeping the Hipparcos distance, the radius of alpha Ara would be unrealistically low (below $3.5 R_{\odot}$ ) or the photosphere unrealistically cold ( $T_{\text {eff }}$ of the order of $\left.15000 \mathrm{~K}\right)$. In Table 5 we show selected parameters of the Be stars alpha Ara, alpha Eri and the "normal" (i.e. non Be) star eta UMa which are all classified as B3 V. Comparing these values, it becomes evident that, if their Hipparcos distances are correct, the striking visual flux differences between these three stars cannot be directly related to a distance difference only.

The reddening of Be stars does not only contain an interstellar term, which is zero for alpha Ara, but also has a circumstellar contribution. This is a model-dependant parameter, explaining the well known difficulty of calibrating the Be stars distances independently of Hipparcos measurements. 
Table 5. Stellar parameters of three close B3V stars, alpha Ara, alpha Eri and eta UMa.

\begin{tabular}{lccc}
\hline \hline & Alpha Ara & Alpha Eri & Eta UMa \\
\hline$V$ & 2.9 & 0.5 & 1.7 \\
Dist. $^{i}$ & $74.3 \pm 6 \mathrm{pc}$ & $44.1 \pm 1.4 \mathrm{pc}$ & $30.9 \pm 0.7 \mathrm{pc}$ \\
$v \sin i$ & $270 \mathrm{~km} \mathrm{~s}^{-1}$ & $225 \mathrm{~km} \mathrm{~s}^{-1}$ & $150 \mathrm{~km} \mathrm{~s}^{-1}$ \\
$B-V$ & -0.17 & -0.17 & -0.17 \\
$V-K^{i i}$ & 0.4 & -0.4 & -0.5 \\
$R_{\text {eq }}{ }^{i i i}$ & $4.8 R_{\odot}$ & $12 \pm 0.4 R_{\odot}$ & $4.8 R_{\odot}$ \\
$R_{\text {pol }}{ }^{i i i}$ & $4.8 R_{\odot}$ & $7.7 \pm 0.2 R_{\odot}$ & $4.8 R_{\odot}$ \\
\hline
\end{tabular}

${ }^{i}$ Hipparcos distance.

${ }^{i i}$ From 2MASS photometry.

iii 4.8 is the statistical value for this B3V star, the radius for alpha Eri is from Domiciano de Souza et al. (2003).

For instance, Cohen et al. (1998) have apparently totally neglected any circumstellar reddening ${ }^{6}$ and derived a distance of $122 \mathrm{pc}$. This difficulty also affects the present model and an underestimation of the circumstellar extinction gives a distance too large. On the other hand, the measured $B-V$ for alpha Ara does not indicate a circumstellar environment dense enough on the line of sight to explain the difference, since this should be linked to emission line properties which are not observed.

A large amount of extinction and reddening of the central star would have to be due to an edge-on disk, and such an inclination would lead to a large and deep self-absorption in the Balmer emission lines, the so-called shell appearance. alpha Ara, however, has never been seen in a shell phase. Instead, the inclination angle is relatively well constrained by the shape of the Balmer line to a value of about $45^{\circ}$.

An inclination of about $45^{\circ}$ implies an equatorial rotational velocity of the order of $380 \mathrm{~km} \mathrm{~s}^{-1}$, since $v \sin i=270 \mathrm{~km} \mathrm{~s}^{-1}$. Using the parameters in Table 3 , this value is at about $75 \%$ of the critical velocity. We point out that the equatorial velocity in the model was considered a free parameter and the best model provides an equatorial value of $300 \mathrm{~km} \mathrm{~s}^{-1}$, i.e. $60 \%$ of the critical velocity. With such a high rotational velocity, the von Zeipel effect becomes important, and thus the continuum emission must be strongly latitude dependant (see e.g. Townsend et al. 2004, his Fig. 3). This may also confuse the line profile diagnostic and affect the angle determination. Such an effect is not included in the present model. A value of the inclination closer to $90^{\circ}$, compatible with the large $v \sin i$ observed, may be related to a stronger circumstellar reddening from the equatorial environment and a lower integrated stellar flux since the equatorial regions of the star are cooler and fainter than the polar ones.

Definitely, the Hipparcos distance, if reliable, is a very constraining parameter for any model of the circumstellar

\footnotetext{
${ }^{6}$ We note also that their estimated distances for other Be stars like $\delta$ Cen seem also systematically overestimated compared to Hipparcos distances. On the contrary their estimated distance of alpha Eri is only $27 \mathrm{pc}$ based on a wrong assumption of the radius of this $\operatorname{star}\left(R_{\mathrm{eq}}=\right.$ $R_{\mathrm{pol}}=4.1 R_{\odot}$ ) which is indeed very difficult to constrain without any interferometric observations.
}

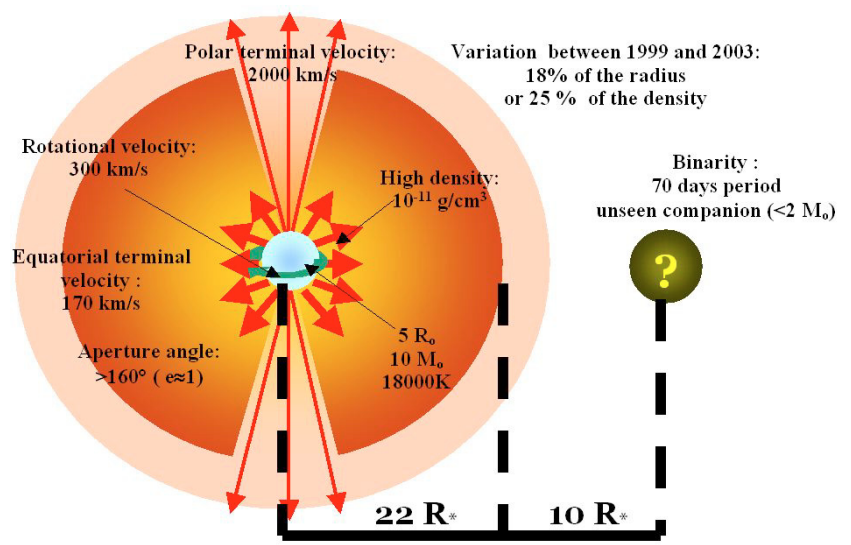

Fig. 13. Schematic view of the alpha Ara circumstellar environment as used in this work (see Table 4).

environment of alpha Ara. More interferometric observations are needed, firstly to constrain the angular size and shape of the hydrogen emission lines, and secondly to evaluate the angular size and shape of the underlying star. The forthcoming VLTI/AMBER instrument will be able to address the first point by studying the fringe properties in $\operatorname{Pa} \beta$ and $\operatorname{Br} \gamma$. However, baselines of the order of $150-200 \mathrm{~m}$ will be needed to resolve the central star, becoming available only with the $1.8 \mathrm{~m}$ Auxiliary Telescopes. Their first light is foreseen for 2005.

\section{Conclusion}

The first IR interferometric measurements of alpha Ara suggest that the size on the circumstellar environment is smaller than what was predicted by Stee (2003). The fact that alpha Ara remains unresolved, but at the same time exhibits a strong Balmer emission line puts very strong constraints on the parameters of its circumstellar disk. Independently of the physical model we used, these measurements put an upper limit of the envelope size in the $N$ band of $\phi_{\max }=4$ mas, i.e. $14 R_{\star}$ if the star is at $74 \mathrm{pc}$ according to the Hipparcos parallax or $20 R_{\star}$ if the star is at $105 \mathrm{pc}$ as suggested by our model, assuming a spherical uniform disk for the star+envelope brightness distribution. The suspected presence of a low mass companion could help to understand the limit of the disk extension as determined by MIDI. A schematic view of the alpha Ara circumstellar disk, as it was modeled in this work with the SIMECA-code, is presented Fig. 13.

Acknowledgements. We thank Anne-Marie Hubert for many fruitful discussions and Jean-Louis Falin for checking the quality of the Hipparcos data. We also thank Anatoly Miroshnichenko for his helpful comments. This research has made use of SIMBAD database, operated at CDS, Strasbourg, France. The paper benefited from the careful reading and advice from the referee, Markus Wittkowski.

\section{References}

Berio, P., Stee, Ph., Vakili, F., et al. 1999, A\&A, 345, 203

Bjorkman, K. S., Nook, M. A., Nordsiek, K. H., et al. 1997, ApJ, 477, 926

van Boekel, R., Verhoelst, T., Leinert, Ch., et al. 2005, A\&A, submitted 
Castor, J. I., Abbott, D. C., \& Klein, R. I. 1975, ApJ, 195, 157

Chauville, J., Zorec, J., Ballereau, D., et al. 2001, A\&A, 378, 861

Cohen, D. H., Cassinelli, J. P., \& MacFarlane, J. J. 1997, ApJ, 487, 867

Coté, J., \& Waters, L. B. F. M. 1987, A\&A, 176, 93

Coté, J., Waters, L. B. F. M., \& Marlborough, J. M. 1996, A\&A, 307, 184

Dachs, J., Engels, D., \& Kiehling, R. 1988, A\&A, 194, 167

Dachs, J., Rohe, D., \& Loose, A. S. 1990, A\&A, 238, 227

Domiciano de Souza, A., Kervella, P., Jankov, S., et al. 2003, A\&A, 407, 2003

Dougherty, S. M., Waters, L. B. F. M., Burki, G., et al. 1994, A\&A, 290, 609

Fabregat, J., \& Reglero, V. 1990, MNRAS, 247, 407

Gehrz, R. D., Hackwell, J. A., \& Jones, T. W. 1974, ApJ, 191, 167

Hanuschik, R. W., Hummel, W., Dietle, O., \& Sutorius, E. 1995, A\&A, 300, 163

Hanuschik, R. W., Hummel, W., Sutorius, E., et al. 1996, A\&AS, 116, 309

Harmanec, P., Habuda, P., Štefl, S., et al. 2000, A\&A, 364, L85

Harmanec, P. 2002, in ASP Conf Ser., 279, 221

Harmanec, Božić, P. H., \& Percy, J. R. 2002, A\&A, 387, 580

Hiltner, W. A., \& Schild, R. E. 1966, ApJ, 143, 770

Hubeny, I. 1988, Comput. Phys. Comm., 52, 103

Hubeny, I., \& Lanz, T. 1995, ApJ, 439, 875

Hummel, M., \& Štefl, S. 2001, A\&A, 368, 471

Koubsky, P., Harmanec, P., Kubat, J., et al. 1997, A\&A, 328, 551

Lamers, H. J. G. L. M., \& Waters, L. B. F. M. 1987, A\&A, 182, 80

Leinert, C., Graser, U., Richichi, A., et al. 2003, The Messenger, 112, 13

Leinert, C., Graser, U., Przygodda, F., et al. 2003, Ap\&SS, 286, 73

Leinert, Ch., van Boekel, R., Waters, L. B. F. M., et al. 2004, A\&A, 423,537

Marlborough, J. M., \& Peters, G. J. 1986, ApJS, 62, 875

Mc Lean, I. S., \& Clarke, D. 1979, MNRAS, 186, 245

Mennickent, R. E. 1989, Rev. Mex. Astron. Astrofis., 19, 107

Mennickent, R. E., \& Vogt, N. 1991, A\&A, 241, 159

Miroshnichenko, A. S., Bjorkman, K. S., \& Krugov, V. D. 2002, PASP, 114,1226
Mourard, D., Bosc, I., Labeyrie, A., Koechlin, L., \& Saha, S. 1989, Nature, 342, 520

Narayanan, V. K., \& Gould, A. 1999, ApJ, 523, 328

Okazaki, A. T. 1997, A\&A, 318, 548

Pan, X., Shao, M., \& Kulkarni, S. R. 2004, Nature, 427, 326

Pickering, E. C., \& Fleming, W. P. 1896, ApJ, 4, 369

Pickering, E. C., \& Cannon, A. J. 1897, ApJ, 6, 349

Poeckert, R., \& Marlborough, J. M., ApJ, 220, 940

Przygodda, F., Chesneau, O., Graser, U., Leinert, C., \& Morel, S. 2003, Ap\&SS, 286, 85

Quirrenbach, A., Buscher, D. F., Mozurkewich, D., et al. 1994, A\&A, 283, L13

Quirrenbach, A., Bjorkman, K. S., Bjorkman, J. E., et al. 1997, ApJ, 479,477

Rivinius, Th., \& Štefl 2000, IAU, 175, 581

Rivinius, Th., Baade, D., \& Štefl, S. 2003, A\&A, 411, 229

Stee, Ph., \& de Araùjo, F. X. 1994, A\&A, 292, 221

Stee, Ph., de Araùjo, F. X., Vakili, F., et al. 1995, A\&A, 300, 219

Stee, Ph., \& Bittar, J. 2001, A\&A, 367, 532

Stee, Ph. 2003, A\&A, 403, 1023

Štefl, S., Hummel, M., \& Rivinius, Th. 2000, A\&A, 358, 208

Thom, C., Granes, P., \& Vakili, F. 1986, A\&A, 165, L13

Townsend, R. H. D., Owocki, S. P., \& Howarth, I. D. 2004, MNRAS, 350,189

Tycner, Ch., Hajian, A. R., Armstrong, J. T., et al. 2004, AJ, 127, 1194

Vakili, F., Mourard, D., Stee, Ph., et al. 1998, A\&A, 335, 261

Waters, L. B. F. M. 1986, A\&A, 162, 121

Waters, L. B. F. M., Coté, J., \& Lamers, H. J. G. L. M. 1987, A\&A, 185,206

Waters, L. B. F. M., \& Marlborough, J. M. 1992, A\&A, 256, 195

Wood, K., Bjorkman, J. E., Whitney, B. A., \& Code, A. D. 1996, ApJ, 461,828

Wood, K., Bjorkman, J. E., Whitney, B. A., \& Code, A. D. 1996, ApJ, 461,847

Yudin, R. V., \& Evans, A. 1998, A\&AS, 131, 401

Yudin, R. V. 2001, A\&A, 368, 912

Zorec, J., \& Briot, D. 1991, A\&A, 245, 150 